\title{
ATENÇÃO FARMACÊUTICA AO IDOSO NA POLIFARMÁCIA
}

\author{
PHARMACEUTICAL ATTENTION TO THE ELDERLY IN POLYPHARMACY
}

\author{
Gabriel Rian dos Santos ${ }^{1}$ \\ Hudson Salles Araújo ${ }^{2}$ \\ Valéria Sobrinho Leal ${ }^{3}$ \\ Douglas Fernando Rambo ${ }^{4}$
}

RESUMO: O profissional farmacêutico está cada vez mais desenvolto em suas habilidades com a população idosa, isso inclui evitar problemas relacionados a medicação, como interações entre os fármacos prescritos pelo médico, reações indesejáveis no tratamento farmacológico, eliminação de complexidade e duplicidade desnecessárias do regime de medicamentos. Por meio dessas atividades o profissional consegue promover um tratamento medicamentoso que seja eficaz e seguro ao seu paciente. Assim, o presente estudo busca revisar a forma em que o profissional farmacêutico pode contribuir na atenção farmacêutica ao idoso na polifarmácia. Objetivo: Revisar sobre a importância da atenção farmacêutica ao uso da polifarmácia em pessoas acima de 60 anos. Métodos: Trata-se de um estudo de revisão de literatura integrativa e de levantamento bibliográfico, cuja a metodologia foi constituída em busca de artigos completos publicados entre os anos de 2009 a 2021 em bases de dados, nas quais foram analisados e selecionados 30 artigos para a elaboração da pesquisa. Utilizou-se como critérios de avaliação para a coleta de informação: assistência farmacêutica, saúde do idoso, interações medicamentosas e polifarmácia. Resultados: Dos 30 artigos, quinze foram escolhidos para

\footnotetext{
I Acadêmico do curso de farmácia da Universidade Salvador (UNIFACS),Salvador-BA. E-mail: grianscruz@hotmail.com.

2 Acadêmico do curso de farmácia da Universidade Salvador (UNIFACS),Salvador-BA. E-mail: Hudsonsalesı45@gmail.com.

3 Acadêmica do curso de farmácia da Universidade Salvador (UNIFACS),Salvador-BA. E-mail: vsobrinholeal@yahoo.com.br.

${ }^{4}$ Graduado Farmacêutico Industrial pela Universidade Regional Integrada do Alto Uruguai e das Missões (2008). Possui Mestrado (2011) e Doutorado (2018) em Ciências Farmacêuticas pela Universidade Federal do Rio Grande do Sul; atuando nas áreas de química de produtos naturais, desenvolvimento e validação de métodos analíticos para controle de qualidade de matéria-prima vegetal. Atuou como colaborador na elaboração /revisão de monografias de plantas medicinais para a Farmacopeia Brasileira (ANVISA). Foi professor na Universidade Regional Integrada do Alto Uruguai e das Missões (Erechim/RS) e no Centro Universitário FADERGS (Porto Alegre/RS). Atualmente é Coordenador do Curso de Farmácia e do Curso de Estética e Cosmética na Universidade Salvador (UNIFACS - BA) e Professor Adjunto da Universidade Federal da Bahia-UFBA. Tem experiência na área de Farmácia, com ênfase em Farmacognosia, Química de Produtos Naturais, Controle de Qualidade, Farmacologia e Cosmetologia.E-mail: Douglas.rambo@unifacs.br.
} 
análise percebeu-se que o uso simultâneo e crônico de medicamentos é cada vez mais comum em idosos, o que intensifica a probabilidade de efeitos colaterais destes, podendo gerar então, uma interação medicamentosa, diminuição da adesão e toxicidade, entre outros. Considerações Finais: A polifarmácia ela tem uma ampla variação no Brasil, sendo utilizada por muitos idosos. Por isso, a atenção farmacêutica é um dos pontos chaves para a utilização da polifarmácia, uma vez que é possível avaliar as prescrições e os medicamentos que estão sendo utilizados de forma inapropriada pelas pessoas.

Palavras-Chave: Atenção farmacêutica, Idosos, Polifarmácia Crônica.

ABSTRACT: This includes avoiding medication-related problems, such as interactions between drugs prescribed by the physician, undesirable reactions in the pharmacological treatment, and elimination of unnecessary complexity and duplication of the drug regimen. Through these activities, the professional is able to promote a drug treatment that is effective and safe for his patient. Thus, the present study aims to review how the pharmaceutical professional can contribute to the pharmaceutical care of the elderly in polypharmacy. Objective: Review over the importance of pharmaceutical care to the use of polypharmacy in people over 6o years of age. Methods: This is a study of integrative literature review and bibliographic survey, whose methodology was constituted in search of complete articles published between the years 2009 to 2021 in databases, in which 30 articles were analyzed and selected for. the elaboration of the research. The following evaluation criteria were used to collect information: pharmaceutical care, health of the elderly, drug interactions and polypharmacy. Results: Of the 30 articles, fifteen were chosen for analysis, it was noticed that the simultaneous and chronic use of medications is increasingly common in the elderly, which intensifies the likelihood of side effects of these, and can then generate a drug interaction, decreased adherence and toxicity, among others. Final Considerations: That polypharmacy has a wide variation in Brazil, being used by many elderly people. Therefore, pharmaceutical care is one of the key points for the use of polypharmacy, since it is possible to evaluate the prescriptions and medicines that are being used inappropriately by people.

Key words: Pharmaceutical care, Elderly, Chronic Polypharmacy.

\section{NTRODUÇÃO}

No contexto social vigente, percebe-se um aumento significativo de pessoas idosas no território brasileiro. O censo populacional mostrou que em 2019, os idosos representavam I3\% da população brasileira (IBGE, 20I8). Para ser considerado idoso, a pessoa deve ter entre 6o anos ou mais. Observa-se que a partir dessa idade, a uma perda significativa da força muscular, aumento da gordura corporal e diminuição da taxa hormonal, no qual ocorre de forma natural e contínua em toda população (MONTIBELLER et al.,20II).

$\mathrm{O}$ processo de envelhecimento do organismo pode influenciar alterações farmacocinéticas, farmacodinâmicas dos fármacos utilizados pelos idosos, isso pode resultar no acúmulo de substâncias tóxicas e dificuldade de eliminação dos metabólitos 
(MOSCA e CORREIA, 20II). Pacientes que apresentam mais de um problema de saúde, são submetidos a utilização de várias drogas, que muitas vezes podem desencadear uma resposta maléfica ao invés do resultado esperado ao seu tratamento. Desta forma a polifarmácia é definida como o uso concomitante de três ou mais medicamentos que são utilizados principalmente pela população idosa. É muito comum que os idosos utilizem a automedicação, por receio de buscar ajuda médica e terem hábitos rotineiros limitados, e com isso ao surgimento de novas doenças, e piora nos quadros clínicos (OLIVEIRA et al., 202I).

A atenção farmacêutica é um ponto chave no processo de assistência farmacêutica e apresenta-se como um medidor de informações para estratégias de saúde, por meio do contato entre o profissional farmacêutico e o paciente. Destarte, é possível reeducá-lo sobre o uso correto dos medicamentos, obter resultados mensuráveis e definidos e promover uma melhora no bem-estar (CORADI, 2012).

O profissional farmacêutico está cada vez mais desenvolto em suas habilidades com a população idosa, isso inclui evitar problemas relacionados a medicação, como interações entre os fármacos prescritos pelo médico, reações indesejáveis no tratamento farmacológico, eliminação de complexidade e duplicidade desnecessárias do regime de medicamentos. Por meio dessas atividades o profissional consegue promover um tratamento medicamentoso que seja eficaz e seguro ao seu paciente. Assim, o presente estudo busca revisar a forma em que o profissional farmacêutico pode contribuir na atenção farmacêutica ao idoso na polifarmácia.

\section{MÉTODOS}

O presente trabalho trata-se de uma revisão de literatura integrativa, de levantamento bibliográfico para busca de informações sobre a importância da polifarmácia aos idosos.

Para a busca dessas informações, utilizou-se as bases de dados eletrônicos: Google acadêmico, Scientific Eletronic Library Online (Scielo) e Lilacs. Os estudos científicos encontrados datam do ano de 2009 a 2021. Usou-se as palavras-chaves: Idosos, atenção farmacêutica e polifarmácia. Encontrou-se 2.360 artigos, com isso, foram analisados os que possuem maior relevância e relatos sobre a importância da atenção farmacêutica ao idoso.

Como critérios de inclusão selecionaram-se artigos, a partir da leitura dos seus resumos, que versassem sobre os descritores. Foram excluídos artigos que não abordavam os 3 descritores. Dentro do período de análise (2010-202I), foram selecionados 30 de acordo com o tema.

Os artigos selecionados, através das bases de dados deverão comprovar a importância da atenção farmacêutica aos idosos e os efeitos da polifarmácia no tratamento medicamentoso. A seguir, é mostrado na figura I, como ocorreu a realização de pesquisa e busca de artigos. 
Figura r: Prospecção da Pesquisa e Busca de artigos:

Utilizaram-se as bases de dados eletrônicos: Google acadêmico, Scientific Eletronic Library Online (Scielo) e Lilacs. Usando as palavraschaves: Idosos, atenção farmacêutica e polifarmácia

Foram selecionados 30 de acordo com o tema.

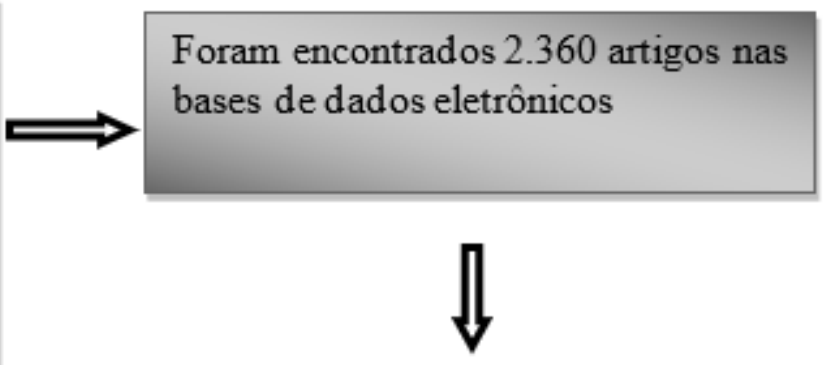

Foram excluídos artigos que não abordavam os 3 descritores. Dentro do período de análise (2009-2021)

\section{RESULTADOS}

Diante da elaboração do trabalho foram selecionados 15 artigos, dentre os quais selecionamos as bases de pesquisa Scientific Eletronic Libray Online (Scielo), Lilacs, Google academico e através da busca de pesquisa, selecionamos de maneira resumida os artigos selecionados para esta revisão em forma de quadro sinóptico Quadro r, contendo os dados dos autores, o tema dos artigos selecionados, os objetivos, os principais resultados e suas conclusões.

Quadro I: Dados científicos, de acordo com o autor, objetivos, principais resultados e conclusões.

\begin{tabular}{|c|c|c|c|c|}
\hline $\mathbf{N}$ & Artigo & Objetivos & Resultados & Conclusões \\
\hline I & $\begin{array}{l}\text { Polifarmácia em idosos: um } \\
\text { estudo com base } \\
\text { populacional. }\end{array}$ & $\begin{array}{l}\text { Investigação da } \\
\text { polifarmácia em } \\
\text { idosos residentes } \\
\text { na área urbana de } \\
\text { Florianópolis (SC) } \\
\text { e seus fatores } \\
\text { associados. }\end{array}$ & $\begin{array}{l}\text { Prevalência de } 32 \%, \text { com } \\
\text { apresentação positiva no sexo } \\
\text { feminino, em que os medicamentos } \\
\text { que foram mais utilizados, eram } \\
\text { indicados para o sistema } \\
\text { cardiovascular, trato alimentar, } \\
\text { metabolismo e sistema nervoso. }\end{array}$ & $\begin{array}{l}\text { Padrão de uso de } \\
\text { medicamentos } \\
\text { está dentro da } \\
\text { média nacional, no } \\
\text { qual a prevalência } \\
\text { da polifarmácia e } \\
\text { as características a } \\
\text { ela foram } \\
\text { semelhantes. }\end{array}$ \\
\hline
\end{tabular}




\begin{tabular}{|c|c|c|c|c|}
\hline 2 & $\begin{array}{l}\text { Consumo de medicamentos } \\
\text { por idosos } \\
\text { Goiânia, Brasil. }\end{array}$ & $\begin{array}{l}\text { Análise de padrão } \\
\text { de consumo de } \\
\text { medicamentos } \\
\text { entre idosos e sua } \\
\text { associação com } \\
\text { aspectos } \\
\text { socioeconômicos e } \\
\text { autopercepção de } \\
\text { saúde. }\end{array}$ & $\begin{array}{l}\text { A prevalência da polifarmácia foi de } \\
26,4 \% \text { e da automedicação de } 35,7 \% \text {. } \\
\text { Os medicamentos mais ingeridos } \\
\text { por automedicação foram os } \\
\text { analgésicos com }(30,8 \%) \text {. Cerca de } \\
24,6 \% \text { dos idosos consumiam } \\
\text { medicamentos considerados } \\
\text { impróprios. Mulheres, viúvos e } \\
\text { idosos com mais de } 80 \text { anos e com } \\
\text { pior autopercepção de saúde } \\
\text { praticavam mais a polifarmácia. }\end{array}$ & $\begin{array}{lr}\text { O padrão do } \\
\text { consumo de } \\
\text { medicamentos por } \\
\text { idosos foi } \\
\text { semelhante ao } \\
\text { encontrado em } \\
\text { outras regiões do } \\
\text { Brasil. O número } \\
\text { de medicamentos } \\
\text { usados, } \\
\text { prevalência das } \\
\text { práticas } \\
\text { polifarmácia } \\
\text { automedicação } \\
\text { consumo } \\
\text { medicamentos } \\
\text { impróprios } \\
\begin{array}{l}\text { estiveram dentro } \\
\text { da média nacional. }\end{array}\end{array}$ \\
\hline 3 & $\begin{array}{l}\text { Prevalência e fatores } \\
\text { associados a potenciais } \\
\text { interações } \\
\text { medicamentosas entre } \\
\text { idosos em um estudo de } \\
\text { base populacional. }\end{array}$ & $\begin{array}{l}\text { Determinar a } \\
\text { prevalência de } \\
\text { potenciais } \\
\text { interações } \\
\text { medicamentosas e } \\
\text { os fatores a elas } \\
\text { associados entre } \\
\text { idosos cadastrados } \\
\text { nas equipes da } \\
\text { Estratégia Saúde da } \\
\text { Família (ESF) do } \\
\text { município de } \\
\text { Timóteo } \\
\text { (MG). }\end{array}$ & $\begin{array}{l}\text { Dos i59 pesquisados, a maioria dos } \\
\text { idosos estava entre 6o a 7o anos de } \\
\text { idade, com renda média familiar de } \\
\text { I,4 a 4,4 salários mínimos, segundo } \\
\text { grau completo, tendo realizado até } \\
\text { três consultas médicas nos últimos I2 } \\
\text { meses. Possuíam doenças crônicas, } \\
\text { sendo hipertensão arterial, diabetes e } \\
\text { colesterol. Quanto ao uso de } \\
\text { medicamentos, a maioria não } \\
\text { praticou automedicação, e a } \\
\text { polifarmácia ocorreu em } 78 \% \text {, sendo } \\
\text { a polifarmácia menor mais } \\
\text { prevalente que a maior. }\end{array}$ & $\begin{array}{l}\text { Devido à grande } \\
\text { frequência de uso } \\
\text { de medicamentos } \\
\text { e suas potenciais } \\
\text { consequências } \\
\text { para a saúde, é } \\
\text { preciso investir na } \\
\text { educação em saúde } \\
\text { visando ao uso } \\
\text { racional de } \\
\text { medicamentos } \\
\text { pelos idosos. }\end{array}$ \\
\hline 4 & $\begin{array}{l}\text { Polifarmácia: interações e } \\
\text { reações adversas no uso de } \\
\text { medicamentos por idosos. }\end{array}$ & $\begin{array}{l}\text { Refletir sobre a } \\
\text { polifarmácia em } \\
\text { idosos com ênfase } \\
\text { nas reações } \\
\text { adversas e nas } \\
\text { interações } \\
\text { medicamentosas. }\end{array}$ & $\begin{array}{l}\text { A vulnerabilidade dos idosos aos } \\
\text { problemas decorrentes do uso de } \\
\text { medicamentos é bastante alta, o que } \\
\text { se deve a complexidade dos } \\
\text { problemas clínicos, à necessidade de } \\
\text { múltiplos agentes terapêuticos e às } \\
\text { alterações farmacocinéticas e } \\
\text { farmacodinâmicas inerentes ao } \\
\text { envelhecimento. }\end{array}$ & $\begin{array}{l}\text { Racionalizar o uso } \\
\text { de medicamentos } \\
\text { e evitar os agravos } \\
\text { advindos da } \\
\text { polifarmácia } \\
\text { serão, sem dúvida, } \\
\text { um dos grandes } \\
\text { desafios da saúde } \\
\text { pública desse } \\
\text { século. }\end{array}$ \\
\hline 5 & $\begin{array}{l}\text { Polifarmácia, doenças } \\
\text { crônicas e marcadores } \\
\text { nutricionais em idosos. }\end{array}$ & 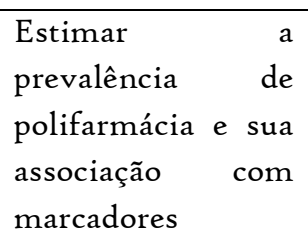 & $\begin{array}{l}\text { A prevalência de polifarmácia foi de } \\
28 \% \text {. Observando-se associação } \\
\text { significativa com sexo feminino; } \\
\text { faixa etária } 75-79 \text { anos; estado } \\
\text { nutricional eutrófico e obeso; uso de }\end{array}$ & $\begin{array}{l}\text { A elevada } \\
\text { prevalência de } \\
\text { polifarmácia e sua } \\
\text { associação com } \\
\text { marcadores }\end{array}$ \\
\hline
\end{tabular}




\begin{tabular}{|c|c|c|c|c|}
\hline & & $\begin{array}{l}\text { nutricionais, } \\
\text { doenças crônicas, } \\
\text { variáveis } \\
\text { sociodemográficas } \\
\text { e de saúde. }\end{array}$ & $\begin{array}{l}\text { dieta, percepção de saúde péssima; } \\
\text { presença de duas, três ou mais } \\
\text { doenças crônicas. }\end{array}$ & $\begin{array}{lr}\text { nutricionais } & \text { e } \\
\text { doenças crônicas } \\
\text { demonstra } \\
\text { necessidade de } \\
\text { vigilância } \\
\text { monitoramento } \\
\text { nutricional em } \\
\text { idosos. }\end{array}$ \\
\hline 6 & $\begin{array}{l}\text { Prevalência e fatores } \\
\text { associados á polifarmácia } \\
\text { em idosos comunitários: } \\
\text { estudo epidemiológico de } \\
\text { base populacional. }\end{array}$ & 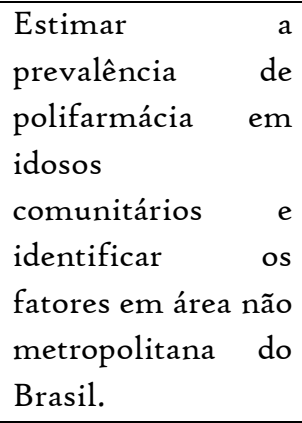 & $\begin{array}{l}\text { Entre os } 686 \text { idosos avaliados, a } \\
\text { prevalência de polifarmácia foi de } \\
23,5 \% \text {. No modelo final } \\
\text { permaneceram como fatores do } \\
\text { desfecho: Hipertensão arterial, } \\
\text { diabetes } \\
\text { mellitus, problemas cardiacos. }\end{array}$ & $\begin{array}{l}\text { Houve elevada } \\
\text { prevalência de } \\
\text { polifarmácia e } \\
\text { importantes } \\
\text { associações com } \\
\text { fatores relativos ás } \\
\text { doenças crônicas. }\end{array}$ \\
\hline 7 & $\begin{array}{lr}\text { USO INAPROPRIADO } \\
\text { DE MEDICAMENTOS } \\
\text { PELO IDOSO: } \\
\text { POLIFARMÁCIA } \\
\text { SEUS EFEITOS }\end{array}$ & $\begin{array}{lr}\text { Identificar } & \text { a } \\
\text { presença } & \text { da } \\
\text { polifarmácia e o } & \text { de } \\
\text { uso } & \\
\text { medicamentos } & \\
\text { potencialmente } \\
\text { inapropriados para } \\
\text { os idosos. }\end{array}$ & $\begin{array}{l}\text { Foram entrevistados } 40 \text { idosos } \\
\text { participantes das atividades do } \\
\text { centro de convivência de } \\
\text { Nanuque/MG, onde } 87,5 \% \text { fazem } \\
\text { uso de medicamentos de uso } \\
\text { contínuo, } 17,5 \% \text { foram classificados } \\
\text { como não } \\
\text { polimedicados,42,5\%classificaram- } \\
\text { se como polifarmácia menor e } 40 \% \\
\text { classificaram-se como polifarmácia } \\
\text { maior. }\end{array}$ & $\begin{array}{l}\text { A ocorrência de } \\
\text { interações } \\
\text { fármaco-fármaco } \\
\text { foi elevada nos } \\
\text { idosos } \\
\text { investigados, } \\
\text { abrangendo } \\
\text { interações } \\
\text { significância } \\
\text { clínica. Ainda, } \\
\text { observou al da } \\
\text { prevalência } \\
\text { utilização } \\
\text { medicamentos } \\
\text { inapropriados em } \\
\text { idosos segundo os } \\
\text { critérios de Beers } \\
\text { 2olz, } \\
\text { comprometendo a } \\
\text { segurança da } \\
\text { farmacoterapia do } \\
\text { idoso, interferindo } \\
\text { significativamente } \\
\text { na funcionalidade } \\
\text { e na qualidade de } \\
\text { vida relacionada à } \\
\text { saúde. }\end{array}$ \\
\hline
\end{tabular}




\begin{tabular}{|c|c|c|c|c|}
\hline 8 & $\begin{array}{l}\text { Atenção farmacêutica ao } \\
\text { idoso: fundamentos } \\
\text { propostas }\end{array}$ & $\begin{array}{l}\text { Apresentar } \\
\text { estratégias } \\
\text { facilitadoras para } \\
\text { implantar o serviço } \\
\text { de Atenção } \\
\text { Farmacêutica, em } \\
\text { farmácia capaz } \\
\text { comunitária, calizar } \\
\text { de ações } \\
\text { cuidados e edanto } \\
\text { educativas, em nível individual } \\
\text { emuanto coletivo, } \\
\text { aos idosos usuários } \\
\text { de medicamentos. }\end{array}$ & $\begin{array}{l}\text { e é possível oferecer atendimento } \\
\text { diferenciado ao idoso com a } \\
\text { implantação da AtenFar e demais } \\
\text { serviços em farmácias comunitárias, } \\
\text { tendo como adicional atividades de } \\
\text { promoção à saúde, possibilitando a } \\
\text { ele compreender a dinâmica do uso } \\
\text { adequado de medicamento, como } \\
\text { também promover a saúde dessa } \\
\text { população usuária. }\end{array}$ & $\begin{array}{l}\text { Estratégias para a } \\
\text { implantação do } \\
\text { serviço de atenção } \\
\text { farmacêutica nas } \\
\text { farmácias } \\
\text { comunitárias são } \\
\text { de suma } \\
\text { importância para } \\
\text { suprir a carência } \\
\text { de informação, no } \\
\text { tocante } \\
\text { medicamentos, em } \\
\text { especial, } \\
\text { população com } \\
\text { faixa da } \\
\text { avançada. etária }\end{array}$ \\
\hline 9 & $\begin{array}{l}\text { Principais } \\
\text { Consequências } \\
\text { Automedicação } \\
\text { Idosos }\end{array}$ & $\begin{array}{lr}\text { Investigar } & \text { na } \\
\text { literatura } & \text { as } \\
\text { principais } & \\
\text { consequências } & \text { da } \\
\text { automedicação em } & \text { em } \\
\text { idosos. }\end{array}$ & $\begin{array}{l}\text { As doenças crônicas acometem mais } \\
\text { os idosos, submetendo-os ao uso } \\
\text { constante de medicamentos. A } \\
\text { reação adversa a um medicamento é } \\
\text { uma resposta ao medicamento de } \\
\text { forma não intencional e prejudicial à } \\
\text { saúde em doses normalmente usadas } \\
\text { pelo ser humano. A interação } \\
\text { medicamentosa ocorre quando há } \\
\text { alguma influência pela ação de outro } \\
\text { medicamento. A automedicação } \\
\text { coloca em risco a saúde da população } \\
\text { idosa. Essa prática pode acentuar os } \\
\text { riscos que estão relacionados aos } \\
\text { medicamentos prescritos, retardar o } \\
\text { diagnóstico adequado e mascarar } \\
\text { uma determinada doença. }\end{array}$ & $\begin{array}{l}\text { Os idosos } \\
\text { constituem } \\
\text { grupo o } \\
\text { vulnerável para o } \\
\text { uso incorreto de } \\
\text { medicamentos, } \\
\text { que pode acarretar } \\
\text { várias } \\
\text { consequências - } \\
\text { orgânicas por meio } \\
\text { das interações } \\
\text { medicamentosas. }\end{array}$ \\
\hline I0 & $\begin{array}{l}\text { IMPORTÂNCIA DO } \\
\text { CUIDADO } \\
\text { FARMACEUTICO AO } \\
\text { PACIENTE IDOSO } \\
\text { QUE FAZ USO DE } \\
\text { POLIFARMÁCIA }\end{array}$ & $\begin{array}{lr}\text { Objetivo desse } \\
\text { estudo é enfatizar a } \\
\text { importância do } \\
\text { cuidado } \\
\text { profissional } \\
\text { farmacêutico em } \\
\text { pacientes idosos } \\
\text { usuários } \\
\text { polifarmácia. }\end{array}$ & $\begin{array}{l}\text { Os resultados obtidos corroboram a } \\
\text { incorporação do cuidado } \\
\text { farmacêutico no serviço público e } \\
\text { privado, que certamente resultaria } \\
\text { em melhores condições de vida e } \\
\text { economia para esses pacientes. }\end{array}$ & 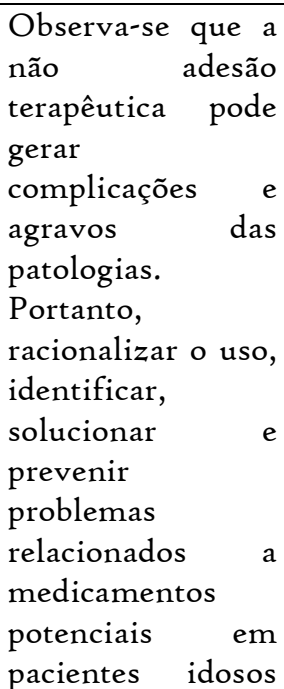 \\
\hline
\end{tabular}




\begin{tabular}{|c|c|c|c|c|}
\hline & & & & $\begin{array}{l}\text { são metas que o } \\
\text { cuidado } \\
\text { farmacêutico } \\
\text { busca alcançar. }\end{array}$ \\
\hline II & $\begin{array}{l}\text { Epidemiologia do uso de } \\
\text { medicamentos entre } \\
\text { idosos em área urbana do } \\
\text { Nordeste do Brasil }\end{array}$ & $\begin{array}{l}\text { Analisar o uso de } \\
\text { medicamentos } \\
\text { entre idosos e os } \\
\text { fatores associados. }\end{array}$ & $\begin{array}{l}\text { A prevalência de uso de } \\
\text { medicamentos foi de } 85,5 \% \text {. A } \\
\text { polifarmácia ocorreu em II\% dos } \\
\text { casos. O uso de polifarmácia } \\
\text { associou-se à escolaridade, à saúde } \\
\text { auto referida, à doença crônica auto } \\
\text { referida e ao número de consultas } \\
\text { médicas ao ano. }\end{array}$ & $\begin{array}{l}\text { A proporção de } \\
\text { uso de } \\
\text { medicamentos é } \\
\text { elevada entre } \\
\text { idosos, inclusive } \\
\text { daqueles } \\
\text { considerados } \\
\text { inadequados, e há } \\
\text { desigualdades } \\
\text { entre grupos de } \\
\text { idosos quando se } \\
\text { considera } \\
\text { escolaridade, } \\
\text { quantidade de } \\
\text { consultas médicas } \\
\text { e saúde auto } \\
\text { referida. }\end{array}$ \\
\hline 12 & $\begin{array}{l}\text { Prevalência e fatores } \\
\text { associados à polifarmácia } \\
\text { entre os idosos residentes } \\
\text { na comunidade }\end{array}$ & $\begin{array}{l}\text { Verificar a } \\
\text { prevalência e os } \\
\text { fatores associados à } \\
\text { polifarmácia entre } \\
\text { os idosos residentes } \\
\text { na comunidade no } \\
\text { município de } \\
\text { Cuiabá, Mato } \\
\text { Grosso. }\end{array}$ & $\begin{array}{l}\text { A prevalência da polifarmácia foi de } \\
\text { ı,30\%. Foram identificadas } \\
\text { associações estatisticamente } \\
\text { significantes entre polifarmácia e } \\
\text { morar acompanhado, ter referido } \\
\text { doenças do aparelho circulatório, } \\
\text { doenças endócrinas, doenças } \\
\text { nutricionais, doença do aparelho } \\
\text { digestivo e ter referido dificuldades } \\
\text { financeiras para aquisição de } \\
\text { medicamentos. }\end{array}$ & $\begin{array}{l}\text { Alguns aspectos } \\
\text { sociais e de } \\
\text { condição de saúde } \\
\text { exercem } \\
\text { importante papel } \\
\text { no uso de } \\
\text { múltiplos } \\
\text { medicamentos } \\
\text { entre os idosos. }\end{array}$ \\
\hline I3 & $\begin{array}{l}\text { PREVALENCIA DA } \\
\text { POLIFARMÁCIA NOS } \\
\text { IDOSOS DE UMA } \\
\text { UNIDADE BÁSICA } \\
\text { DE SAÚDE NO } \\
\text { ESTADO DE MINAS } \\
\text { GERAIS }\end{array}$ & $\begin{array}{l}\text { Analisar a } \\
\text { prevalência da } \\
\text { polifarmácia em } \\
\text { idosos de uma } \\
\text { unidade básica de } \\
\text { saúde (UBS), } \\
\text { correlacionando } \\
\text { com o gênero e a } \\
\text { faixa etária. }\end{array}$ & $\begin{array}{l}\text { De acordo com as análises, dos } 282 \\
\text { participantes, } 40,4 \% \text { encontravam-se } \\
\text { em polifarmácia, sendo que } 45,2 \% \\
\text { eram mulheres e apenas } 31,25 \% \text { eram } \\
\text { homens. A pesquisa também } \\
\text { mostrou uma relação significativa } \\
\text { entre polifarmácia e faixa etária, } \\
\text { onde 31,09\% dos idosos entre } 60 \text { e } 69 \\
\text { anos encontravam-se em } \\
\text { polifarmácia, } 46,49 \% \text { dos entre } 70 \text { e } \\
79 \text { anos e } 48,97 \% \text { dos entre } 80 \text { e } 89 \\
\text { anos. }\end{array}$ & $\begin{array}{l}\text { Os dados } \\
\text { analisados } \\
\text { evidenciam } \\
\text { relação entre o } \\
\text { gênero feminino } \\
\text { com } \\
\text { polifarmácia, ou } \\
\text { seja, ser idosa no } \\
\text { estudo constituiu } \\
\text { um fator de risco } \\
\text { para polifarmácia. }\end{array}$ \\
\hline I4 & $\begin{array}{l}\text { MULTIMORBIDADE } \\
\text { E POLIFARMÁCIA } \\
\text { EM } \\
\text { RESIDENTES IDODOS } \\
\text { COMUNIDADE }\end{array}$ & $\begin{array}{l}\text { Identificara } \\
\text { prevalência da } \\
\text { multimorbidade e } \\
\text { polifarmácia em } \\
\text { idosos. }\end{array}$ & $\begin{array}{l}\text { A idade variou de } 60 \text { a } 90 \text { anos, com } \\
\text { média de } 69,3 \quad( \pm 5,47) \text {. O sexo } \\
\text { feminino prevaleceu com } 78,0 \% \text {. O } \\
\text { total de multimorbidade variou de } \\
\text { duas a nove condições crônicas, com } \\
\text { prevalência de } 75,0 \% \text { de idosos com }\end{array}$ & $\begin{array}{l}\text { Entre os idosos } \\
\text { que possuíam } \\
\text { multimorbidade e } \\
\text { polifarmácia } \\
\text { prevaleceram as } \\
\text { mulheres } \\
\text { pertencentes à }\end{array}$ \\
\hline
\end{tabular}




\begin{tabular}{|c|c|c|c|c|}
\hline & & & $\begin{array}{l}\text { multimorbidade. As condições } \\
\text { crônicas mais referidas foram } \\
\text { Hipertensão Arterial, com } 76,0 \% \text { e } \\
\text { Diabetes Mellitus, com 46,0\%. O uso } \\
\text { de medicação continua está presente } \\
\text { em 86,o\% dos idosos e a prevalência } \\
\text { de polifarmácia foi de I8,o\%. }\end{array}$ & $\begin{array}{l}\text { classe econômica } \\
\text { mais pobre. }\end{array}$ \\
\hline 15 & $\begin{array}{l}\text { Assistência farmacêutica } \\
\text { em casos de polifarmácia } \\
\text { entre a população idosa }\end{array}$ & $\begin{array}{l}\text { O presente estudo } \\
\text { teve como objetivo } \\
\text { discutir as } \\
\text { possíveis } \\
\text { contribuições da } \\
\text { assistência } \\
\text { farmacêutica para o } \\
\text { paciente idoso poli } \\
\text { medicado. }\end{array}$ & $\begin{array}{l}\text { A alta prevalência da polifarmácia e } \\
\text { do uso de medicamentos } \\
\text { potencialmente inapropriados entre } \\
\text { os idosos. Variáveis como morar } \\
\text { sozinho, baixa escolaridade, estado } \\
\text { civil, sexo, idade, presença de } \\
\text { doenças crônicas interferem } \\
\text { negativamente na qualidade de vida } \\
\text { do idoso. }\end{array}$ & \begin{tabular}{lr} 
A & \multicolumn{2}{c}{ assistência } \\
farmacêutica é \\
uma alternativa \\
eficaz e de suma \\
importância na \\
obtenção \\
melhoria da \\
qualidade de vida \\
do idoso, \\
reduzindo \\
impactos da \\
morbimortalidade \\
relacionada ao uso \\
de vários \\
medicamentos.
\end{tabular} \\
\hline
\end{tabular}

Fonte: Autores (2021).

DISCUSSÃO

Em vista dos resultados apresentados; algumas categorias de discussão foram criadas, a saber:

\section{A polifarmácia em idosos com variados contextos}

A população idosa é um grupo pertencente de pessoas que mais crescem no Brasil, e são os maiores consumidores de medicamentos no mundo, devido a esse grande consumo, o Pais ocupa a sexta posição de mercado mundial com maior consumo de medicamentos (OLIVEIRA E CORRADI,2018). Portanto o processo de envelhecimento está associado as doenças cronicas degenerativas que é fator para uma maior demanda aos serviços de saúde. Fatores como tabagismo, consumo excessivo de álcool, excesso de peso favorece o surgimento de doenças cronicas que são mais conhecidas como DCNT (doenças cronicas não transmissíveis) que tem maior incidências em pessoas nessa faixa etária, com isso faz com que essas pessoas utilizem medicamentos de uso continuo. Um elevado consumo de medicamentos afeta negativamente a qualidade de vida do idoso, porém esses mesmos medicamentos são os que ajudam a prorrogar a vida, deste modo a polifarmácia não é necessariamente á que apresenta potenciais de riscos para eventos adversos, mais sim o seu uso indevido (ALMEIDA et al.,2017).

O uso simultâneo e crônico de medicamentos é cada vez mais comum em idosos, uma vez que intensifica a probabilidade de efeitos colaterais, a qual pode gerar uma interação medicamentosa, diminuição da adesão do fármaco e toxicidade, entre outras problemáticas. Os idosos são expostos a consequências do uso de múltiplos medicamentos, estes, podem apresentar alterações fisiológicas que modificam a farmacodinâmica e a farmacocinética, 
contribuindo assim, para a toxicidade. As interações entre medicamentos são alterações na ação desses, devido à ingestão simultânea de outro fármaco ou alimento. Essas interações podem reduzir ou aumentar o efeito de um medicamento, o que pode ter uma implicação imprevisível no tratamento. Os erros ao uso excessivo de medicamentos estão relacionado as mais comuns utilizações, nas quais envolvem, medicamentos impróprios, dose erradas, frequência inadequada, período insuficiente ou demasiado de consumo, além de outras combinações com fármacos, ocasionando interações indesejadas (MUNIZ et al.,2017). Portanto, o profissional de saúde tem que ficar atento em relação a prescrição de múltiplos medicamentos aos idosos, para evitar danos à saúde e à segurança deles (SECOLI, 20Io).

A automedição é uma cultura médico-farmacêutica onde sua pratica não é restrita no Brasil.Com o surgimento da covid-I9 no Pais, percebeu-se um aumento significativo na procura de medicamentos sem comprovação cientifica,pois,essas idas e vindas do vírus SARS-CoV-2, mas conhecido como COVID-19, acabaram gerando uma certa ansiedade nos individuos, fazendo com que, ocorra a automedicação ou acabar evoluindo para vários kits de medicamentos, que consequentemente não são indicados e muito menos sem comprovação cientifica de eficácia, portanto o individuo acaba ficando desprotegido na fase inflamatória (MURREK ,202I).Logo Os profissionais de saúde, em especial os farmacêuticos, eles precisam orientar, a ter um cuidado a mais em relação ao uso racional de medicamentos, de forma abrangente, fazendo implementações para auxiliar em uma excelente segurança ao paciente deste(BISPO,2020).

\section{A importância da atenção farmacêutica em idosos na polifarmácia}

A atenção farmacêutica é um modelo de prática que surgiu com o intuito de garantir ao paciente uma farmacoterapia racional, com segurança e com um menor custo. Por meio de ações educacionais, o profissional farmacêutico consegue aconselhar o paciente sobre o uso racional de medicamentos, a forma de utilização, sua posologia e seus horários adequados para uma melhor adesão (FIDÊNCIO e YAMACITA, 20II).O farmacêutico é um profissional qualificado para intervir com qualquer irregularidade medicamentosa, aconselhando o paciente ou até mesmo intervir em receitas, mediante de processos bem documentados, para evitar erros de prescrições e dosagens de medicamentos (FARIAS et al.,2018). No decorrer, pacientes idosos que apresentam múltiplas patologias têm a tendência de utilização de múltiplos medicamentos (polifarmácia); erros de administração; falhas nas aderências dos regimes terapêuticos, que aumentam com o avanço da idade, faz com que o desvio padrão da farmacocinética e da farmacodinâmica, ocorrem com mais frequências nesses pacientes do que em jovens (MENEZES e SÁ, 2010).

Diante das estratégias e recomendações à atenção farmacêutica ao idoso, é de suma importância, já que irá suprir a carência de informações a respeito dos medicamentos prescritos nessa faixa etária. Para uma melhor adesão á terapia medicamentosa, em decorrência aos resultados clínicos indispensável os serviços de acompanhamento 
farmacêutico, como consultas para organização de esquemas de cuidados, resolver os impasses relativos aos medicamentos e proporcionar acompanhamento congruente, com foco na aquisição de habilidades e competências para a corresponsabilização (NASCIMENTO et al.,2017). O aconselhamento aos doentes acerca do uso de medicamentos de venda livre, e a participação em programas de educação para a saúde em colaboração com outros membros da equipe de saúde são algumas estratégias de como a atenção farmacêutica pode ajudar a população idosa (MARQUES et al.,2017). Contudo, deve-se repensar sobre o papel do farmacêutico na saúde e a importância da implementação do serviço de atenção farmacêutica nas farmácias comunitárias (MENEZES e SÁ, 20ıo).

\section{Analisar o papel do profissional farmacêutico na saúde do idoso.}

O profissional farmacêutico, por meio do seu conhecimento sobre os fármacos, consegue proporcionar uma melhor terapia racional ao uso correto dos medicamentos, portanto ele pode proporcionar informações privilegiadas e seguranças ao paciente, como, posologia, formas de utilização do fármaco que foi prescrito, melhores horários para a administração desses fármacos, além de esclarecer duvidas referentes a qual classe terapêutica pertence. Através dessas estratégias o farmacêutico tem como objetivo proporcionar um plano terapêutico eficaz (PINTO et al., 2013). Saber o perfil dos idosos, permite-se planejar os cuidados farmacêuticos mais adequados e encaminhar para as intervenções específicas na realização do seu tratamento (SALES et al.,2017). A analise do profissional farmacêutico ao paciente tem como finalidade de promover um tratamento medicamentoso eficaz e seguro, portanto a sua presença e de suma importância pois consegue proporcionar o uso racional do medicamento e, consequentemente, informar a população sobre esta importância. $O$ profissional farmacêutico tem o direito de intervir na questão de receitas medicamentosas, com o intuito de auxiliar para um tratamento medicamentoso seguro e eficaz, mediado na orientação com os pacientes e familiares.

O farmacêutico possui importantes atividades relacionadas a melhor qualidade de vida para os idosos, sendo elas: realizar o acompanhamento farmacoterapêutico, a verificação da farmacoterapia para avaliar se os medicamentos prescritos são necessários, eficazes e seguros, bem como proporcionar adesão à terapia; supervisionar efeitos adversos; efetuar acompanhamento em saúde, a fim de investigar de problemas de saúde, como diabetes, dislipidemias e hipertensão arterial; proporcionar atividades de educação em saúde relacionada ao uso racional de medicamentos e à prevenção de doenças e orientações farmacêuticas em geral.

\section{CONSIDERAÇÕES FINAIS}

O presente estudo teve como foco a polifarmácia em idosos. Com isso, foi possível perceber que a polifarmácia tem uma ampla variação no Brasil, sendo utilizada por muitos idosos. Os artigos que foram selecionados prevaleceram e investigaram o uso da polifarmácia em diferentes grupos de idosos. Apesar de não ter sido utilizado nos resultados dos artigos selecionados, a atenção farmacêutica é um dos pontos chaves para a utilização 
da polifarmácia. Por meio dela é possível avaliar as prescrições e os medicamentos que estão sendo utilizados de forma inapropriada pelas pessoas. Logo, novas produções cientificas nas quais sejam destacadas a importância do profissional farmacêutico na atenção aos idosos, principalmente na polifarmácia, são necessárias.

\section{REFERÊNCIAS}

ALMEIDA, Natália Araujo et al.Prevalência e fatores associados á polifarmácia entre os idosos residentes na comunidade. Revista brasileira geriatria e gerontologia. 2017. Disponível em https://www.scielo.br/j/rbgg/a/n5vypZTvfYhhYJxPdYr7Dbb/?lang=pt acesso em :Io de janeiro de 202I.

ARAUJO,Camila Soares et al. Importância do cuidado farmacêutico ao paciente idoso que faz uso de polifarmácia. Editora Realiza, 2019.Disponível em: https://www.editorarealize.com.br/editora/anais/cieh/2019/TRABALHO_EVI25_MDI _SA3_IDio57_03062019182420.pdf acesso em: 20 de janeiro de 2021.

BARBOSA,Maira Tonidandel. Os idosos e a complexidade dos regimes terapêuticos. Revista da Associação Medica Brasileira, 2009.Disponível em: https://www.scielo.br/j/ramb/a/tksXq8TQ5q7G4xvKFPVc6WS/?lang=pt acesso em: o5 de fevereiro de 202I.

BISPO,Jessé Eduardo. Automedicação e os riscos á saúde em tempos de covid-I9, 2020. Disponível em : https://eephcfmusp.org.br/portal/online/automedicacao-riscos-saudecovidig/ acesso em :Io de fevereiro 2021.

BONGIOVANI,Lucimara Fátima Lopes de Andrade. Multimorbidade e polifarmácia em idosos residentes comunidade. Revista online de pesquisa cuidado é fundamental, 2021.Disponível em : http://www.seer.unirio.br/cuidadofundamental/article/view/8644 acesso em:II de fevereiro de 202I.

CARDOSO,Daiane Manoelina;PILOTO,Juliana Antunes Da Rocha. Atenção farmacêutica ao idoso: uma revisão. Brazilian Journal of Surgery and Clinical Research -BJSCR, Maringá-PR, 2014. Disponível em: https://www.mastereditora.com.br/periodico/2014I130_215818.pdf acesso em:13 de fevereiro de 2021.

CIVINSKI,Cristian;André Montibeller;OLIVEIRA,André Luiz. A importância do exercício físico no envelhecimento. Revista da UNIFEBE, 20II. Disponível em: https://periodicos.unifebe.edu.br/index.php/revistaeletronicadaunifebe/article/view/6 8 acesso em:I2 de abril de 2021.

CORADI,Ana Elisa Prado. A importância do farmacêutico no ciclo da Assistência Farmacêutica. Arquivos Brasileiros de Ciências da Saúde, v.37, n. 2,2012; p. 62-64. Disponível em: http://files.bvs.br/upload/S/1983-2451/2012/v37n2/a3051.pdf acesso em:I5 de fevereiro de 2021.

DANTAS,Michelle Silva;SANTOS,Vanessa Cruz. Implicações da polifarmácia entre idosos e a contribuição da atenção farmacêutica. Lecturas: Educación Física y Deportes, 2018.

Disponível em: 
https://www.efdeportes.com/efdeportes/index.php/EFDeportes/article/download/273 $/$ I73 ?inline $=\mathrm{I}$ acesso em:I6 de fevereiro de 2021.

GALATO,Dayani; SILVA, Eduarda Souza; TIBURCIO,Leticia de Souza. Estudo de utilização de medicamentos em idosos residentes em uma cidade do sul de Santa Catarina (Brasil): um olhar sobre a polimedicação. Ciência \& Saúde Coletiva, 20 o. Disponível em https://www.scielo.br/j/csc/a/BmGLqzQzZRpBF5pR3mzFz9G/abstract/?lang=pt acesso em: ir de Março de 202I.

GORTADELO,Daniel Riani et al. Prevalência e fatores associados a potenciais interações medicamentosas entre idosos em um estudo de base populacional. Revista Brasileira de Medicina de Família e Comunidade, v.9, n.31, 2014. Disponível em: https://rbmfc.org.br/rbmfc/article/view/833 acesso em :1o de abril de 2021.

MENEZES,André Luis Lima;SÁ, Maria Lúcia Barreto. Atenção farmacêutica ao idoso: fundamentos e propostas. Revista Brasileira de Geriatria e Gerontologia, 2010.Disponível em: https://cdn.publisher.gni.link/ggaging.com/pdf/v4n3ao7.pdf acesso em:o6 de maio de 2021.

MOSCA, Carolina;CORREIA ,Paula. O medicamento no doente idoso. Acta Farmacêutica Portuguesa, 2012.Disponível em: https://www.actafarmaceuticaportuguesa.com/index.php/afp/article/view/23 acesso em: I2 de abril de 202I.

MUNICZ,Elaine Cristina Salzedas et al. Análise do uso de medicamentos por idosos usuários de plano de saúde suplementar. Revista Brasileira de geriatria e gerontologia.2017.Disponível em: https://www.scielo.br/j/rbgg/a/KnHxGZJftzLoCygQMWV37hM/?lang=pt acesso em :I4 de abril de 2021.

NEVES ,Sabrina Joany Felizando et al. Epidemiologia do uso de medicamentos entre idosos em área urbana do Nordeste do Brasil. Revista saúde publica, 2013. Disponível em : https://www.scielo.br/j/rsp/a/DdCFNYznn8tw6Rr8pBs48GD/abstract/?lang=pt acesso em:04 de maio de 2021.

OLIVEIRA,Patricia Carvalho et al. Prevalência e

fatores associados à polifarmácia em idosos atendidos na atenção primária à saúde em Belo Horizonte-MG, Brasil. Ciência \& Saúde Coletiva, 2021. Disponível em: https://www.cienciaesaudecoletiva.com.br/artigos/prevalencia-e-fatores-associados-apolifarmacia-em-idosos-atendidos-na-atencao-primaria-a-saude-em-belo-horizonte$\mathrm{mg} / \mathrm{r} 7294$ ? $\mathrm{id}=\mathrm{I} 7294 \& \mathrm{id}=\mathrm{I} 7294$ acesso $\mathrm{em}: 02$ de março de 2021.

PEREIRA, Karine Gonçalves et al .Polifarmácia em idosos: um

estudo de base populacional. Revista Brasil Epidemiol, Florianópolis-SC, 2017. Disponível em : https://www.scielo.br/j/rbepid/a/HW5m6chDzrqRpMh8xJVvDrx/?lang=pt acesso em :18 de abril de 2021.

PERISSÉ, Camille;MARLI, Mônica. Idosos indicam caminhos para uma melhor idade. Revista Retratos, 2019. Disponível em: https://agenciadenoticias.ibge.gov.br/agencia- 
noticias/2012-agencia-de-noticias/noticias/24036-idosos-indicam-caminhos-para-umamelhor-idade acesso em:19 de abril de 2021.

PINTO, Isabela Voz Leite; CASTRO,Mariza dos Santos; REIS;Adriano Max Moreira. Descrição da atuação do farmacêutico em equipe multiprofissional com ênfase no cuidado ao idoso hospitalizado. Revista Brasileira de Geriatria e Gerontologia, v.16 n.4, Rio de Janeiro, 2013. Disponível em: https://www.scielo.br/pdf/rbgg/vi6n4/r8o9-9823-rbgg-ı604-00747.pdf acesso em: 20 de abril de 2021.

RIBAS, Carlise; OLIVEIRA,Karla Renata. Perfil dos medicamentos prescritos para idosos em uma Unidade Básica de Saúde do município de Ijuí-RS. Revista Brasileira de Geriatria e Gerontologia, 2014.Disponível em: https://www.scielo.br/j/rbgg/a/NYhPHzBrfsFGTNYn87S6cnz/abstract/?lang=pt acesso em:2I de abril de 2021.

ROSA,Gabriela Rotello; CAMARGO, Eliana Anunciato Franco. Polimedicação em idosos. Revista Interciência \& Sociedade, 2014. Disponível em: https://intercienciaesociedade.francomontoro.com.br/colecao/impressa/v3_n2/polimed icacao_idosos.pdf acesso em:03 de maio de 2021.

SANTOS, Thalyta Renata Araújo et al. Consumo de medicamentos por idosos. Revista de Saúde Pública, Goiânia-GO, 2012. Disponível em: https://www.scielo.br/pdf/rsp/v47nI/r3.pdf acesso em:24 de abril de 2021.

SALES ,Alessandra Santos;SALES, Marta Gabriele Santos; CASOTTI ,Cezar Augusto. Perfil farmacoterapêutico e fatores associados á polifarmácia entre idosos de Aiquara Bahia. V.26, p.121-132, 2017.Disponível em: https://www.scielo.br/j/ress/a/kDxqZTspWMgfT 4 Yxx93dC9g/abstract/?lang=pt acesso em:25 de abril de 2021.

SECOLI, Silvia Regina. Polifarmácia: interações e reações adversas no uso de medicamentos por idosos. Revista Brasileira de Enfermagem, v.63, n.I, Brasília-DF, 2010.Disponível em: https://www.scielo.br/pdf/reben/v63nI/v63nia23.pdf acesso em:26 de abril de 202I.

SILVA,Elaine Aparecida;CONCI ML. Polifarmácia em Idosos. Revista Saúde e Pesquisa, setembro, 2013.Disponivel em : https://periodicos.unicesumar.edu.br/index.php/saudpesq/article/view/2862 acesso em:or de Janeiro de 2021.

SILVEIRA, Erika Aparecida; DALASTRA ,Luana; PAGOTTO, Valéria. Polifarmácia, doenças crônicas e marcadores nutricionais em idosos. Revista Brasileira de Epidemiologia, 2014.Disponível em: https://www.scielo.br/pdf/rbepid/vi7n4/pt_I4I5790X-rbepid-17-04-00818.pdf acesso em:20 de abril de 2021.

SILVEIRA ,Priscila Assis; SILVA, Samuel Campos; ROCHA, Karine Siqueira Cabral. Prevalência da polifarmácia nos idosos de uma unidade básica de saúde no estado de Minas Gerais. Revista atenção saúde v.16 n.58, p.29-35, 2018. Disponivel em: https://seer.uscs.edu.br/index.php/revista_ciencias_saude/article/view/5364 acesso em:05 de março de 2021. 
SILVA,Anne Caroline Araújo et al. Assistência farmacêutica em casos de polifarmácia entre a população idosa. Revista Eletrônica Acervo Saúde, 2019.Disponível em: https://acervomais.com.br/index.php/saude/article/view/999 acesso em:22 de março de 2021.

SOUZA, Dayana Matos et al. Uso inapropriado de

medicamentos pelo idoso: polifarmácia e seus efeitos. Pensar Acadêmico (facig.edu.br), 2018.Disponível em: http://pensaracademico.facig.edu.br/index.php/pensaracademico/article/view/36r acesso em:I5 de março de 2021.

GALVÃO, Zelia. Atenção farmacêutica ao idoso: uma proposta para a continuidade do tratamento,2018. em :http://revista.oswaldocruz.br/Content/pdf/Edicao_I8_Zelia_Galv\%C3\%A3o.pdf acesso em:or de maio de 202i. 